\title{
Using Machine Learning and Artificial Intelligence Principles to Implement a Wealth Management System
}

\author{
Prarthana Mukherjee, Prit Palan, Mohan V Bonde
}

\begin{abstract}
Studies have shown that new generation of millennials have limited to no knowledge about managing their finances. This lack of awareness has created a need for financial literacy which is not only an essential employ-ability skill but also, a paramount life skill. Not only the younger generation but many individuals already in the corporate field are at their wit's end when it comes to planning their finances and making correct financial decisions. This is where awareness in wealth management comes in. Wealth management is an investment advisory service. It also combines financial services to address the needs of individuals. It is more than just investment advice; it encompasses all parts of a person's financial life. The users can find all the information of different investments rather than integrating all the information from different places. They can generate a plan themselves or with the help of artificial intelligence and machine learning principles, manage their own and their family's current and future needs.
\end{abstract}

Keywords: Artificial Intelligence, Investment Advisory, Machine Learning, Wealth Management System

\section{INTRODUCTION}

$\mathrm{W}$ ealth management is an investment advisory service. It combines many financial services to cater to the needs of the clients. In this process the advisor understands and anticipates the client's wants and creates a bespoke strategy using appropriate financial products and services. For such financial advice mostly, people pay a certain sum to a wealth management advisor or a wealth manager. Depending upon client's financial status the advisor suggests how much should the client invest and in which investments. Wealth management is more than just investment advice. It also can encompass all parts of an individual's financial life.

Manuscript received on May 21, 2021

Revised Manuscript received on May 27, 2021.

Manuscript published on May 30, 2021.

* Correspondence Author

Prarthana Mukherjee*, Pursuing Bachelor of Engineering, Department Information Technology, Usha Mittal Institute of Technology, SNDT Women's University, Mumbai, Maharashtra, India.

Prit Palan, Pursuing Bachelor of Engineering, Department Information Technology, Usha Mittal Institute of Technology, SNDT Women's University, Mumbai, Maharashtra, India.

M. V. Bonde, Assistant Professor, Department Information Technology, Usha Mittal Institute of Technology, SNDT Women's University, Mumbai, Maharashtra, India

(C) The Authors. Published by Blue Eyes Intelligence Engineering and Sciences Publication (BEIESP). This is an open access article under the CC BY-NC-ND license (http://creativecommons.org/licenses/by-nc-nd/4.0/)
A user of this system can find all the information of different investments, instead of piecing it together from a number of places. They can generate a plan themselves manually or with the help of artificial intelligence and machine learning techniques and manage their own and their family's current and future needs. However, it is a common misnomer that wealth management is a service required and available only to wealthy individuals. In fact, with new world of working millennial generation, there is a growing need appropriate guidance and understanding into the finance world before they can truly delve into it. In this website, we have created a user-friendly platform such that individuals aren't intimidated by financial world and the surplus of information around.

We have built a website where any individual, as a user will be able to understand the various investment options and opportunities available. They will be able to make accurate financial decisions with the help of various features like returns calculators, financial plan creators, stock market predictors, and stock market simulators.

\section{A. Objectives of the Study}

In today's world technology plays a very important role. The main objective of the wealth management system is to ease managing an individual's finances and to create a platform where users can make well informed and best suited financial decisions. The system is based on the user's input information regarding the necessary parameters which are important to accurately calculate and provide best suited options for investing.

We have used various machine learning and artificial intelligence principles to accurately allot an individual into the categories of high risk, medium risk and low risk. Based on their category of risk, the user will be provided with various financial plans and calculators to understand the approximate returns on those investments, should they decide to go for them. Above all, we aim to provide a comfortable user experience along with gained expertise in the financial literacy field.

\section{B. Organization of the Paper}

- Related Work: We looked into the various existing systems similar to the proposed system to ascertain the important features that are to be worked on.

- Proposed Methodology: The proposed system design and module division are discussed to understand the system better.

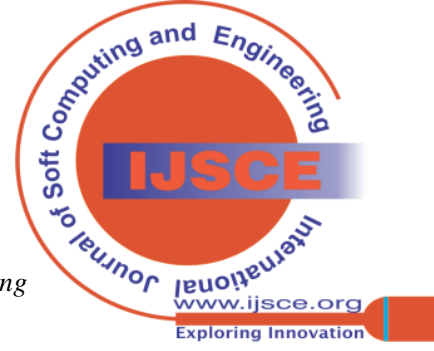


- Results and Discussion: Along with installation and testing of the software that are used for development of the system. As well as the various observations during the implementation.

- Conclusion and Future scope of the system.

\section{RELATED WORK}

There are a number of existing works which are based on similar aspects of wealth management. These works are listed and reviewed for ascertaining the main features which must be included in the system. Also to determine the advantages that could be added into the proposed system to further benefit the user and in all improve the system. A few works are as followed:

\section{A. Stock Market Analysis and Prediction Co- Authored by Eric Alexander, Emily Kawaler}

Through this paper we learnt that the stock market data is influenced by a large number of factors including foreign and domestic economies, trade agreements, wars, seasons, and even day of the week. Many different approaches towards prediction are attempted in this paper including neural networks and fuzzy reasoning, support vector machines, and even attempting prediction using data-mining techniques over textual data in financial news. In this paper, we were able to ascertain the abilities of linear regression, random forests, and support vector machines (SVM) with SMO to predict future prices and trends in a variety of stocks.

\section{B. Study on Machine Learning Techniques in Financial Markets o-Authored by Prakhar Vats, Krishna Samdani}

This Paper helped understand the financial world and its pillars, portfolios, securities, stock market forecasting, risk management, debt management, and these pillars rely on adequate and accurate prediction. These are problems that, on the small scale, affect individuals and their financial conditions and, on the larger scale, can be detrimental to a country's or an organization's financial well-being. This paper, implements various algorithms and platforms involving Machine Learning in Financial Markets and reaching informed conclusions on parameters like accuracy, efficiency, speed and usability. This paper, helped us decide upon the algorithms to use in the proposed system based on the parameters they have tested.

\section{Finbingo}

Finbingo is an application which was recently launched in the market as a financial decision helper. It enables the users to ascertain their financial goals in terms of money or objects. It also enables the users to understand and ascertain the financial plan to achieve that goal. It helps the user understand the various investment plans which can enable them to invest accurately. We understood the need for high connectivity with users and the need to be easily accessible by the users.

\section{PROPOSED METHODOLOGY}

We have split the entire system into four distinct modules based on the risk level of the investment options and the customer's view to the system, they are as followed:

\section{A. Customer Module:}

The first module of the system is the customer module, through this module the user will enter in their individual details and other details regarding their earnings, expenses and savings. Based on these details the system create the financial plans for the individuals using artificial intelligence principles.

\section{B. Low-risk and Medium-risk Module}

The second module includes low and medium risk investment. In low-risk investment users can invest and can be assured that their money is safe. They include investments like fixed deposits and PPfs. The medium risk investments include mutual funds. It will also include calculators to calculate their returns based on the investment they are interested in.

\section{High-risk Module}

This module includes high risk investments like the stock market. In this module users will be displayed the various stocks which are on the rise and have a probability of an increased profit. These predictions will be done using machine learning principles. The user may also individually search any stock they are interested in to find their predictions.

\section{Integration Module}

In this module, all the above mentioned modules are integrated and the website is built. In this module the front end implementation and development of system is done.

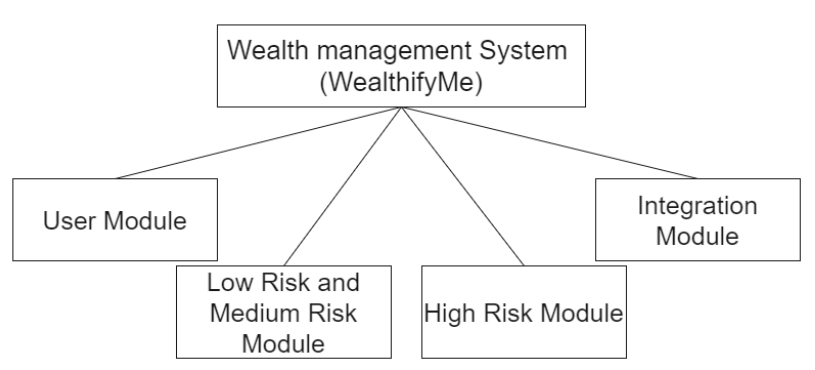

Fig 1: Block view of the System

\section{IMPLEMENTATION}

\section{A. Website Implementation}

React is a user interface library written in JavaScript. Single-page applications are built with React. ReactJS is a JavaScript library for creating reusable user interface components that is declarative, powerful, and versatile. It's an open-source, component-based front-end library that's only liable for the application's view layer. We can make reusable UI components with React. Developers can use React to build massive web applications that can alter data without reloading the page. React's main goal is to be fast, scalable, and easy to use. It only operates on the application's user interfaces.
Published By:

Blue Eyes Intelligence Engineering and Sciences Publication

(C) Copyright: All rights reserved.

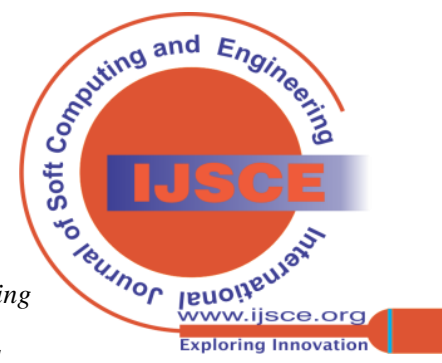


This corresponds to the MVC template's view. As a user enters into the website, they will view the home page, if they are not a registered user, they can register themselves in the sign-up section. As a new user, a user needs to fill out a form about the various information that would be necessary to segregate them into the various level of risk.

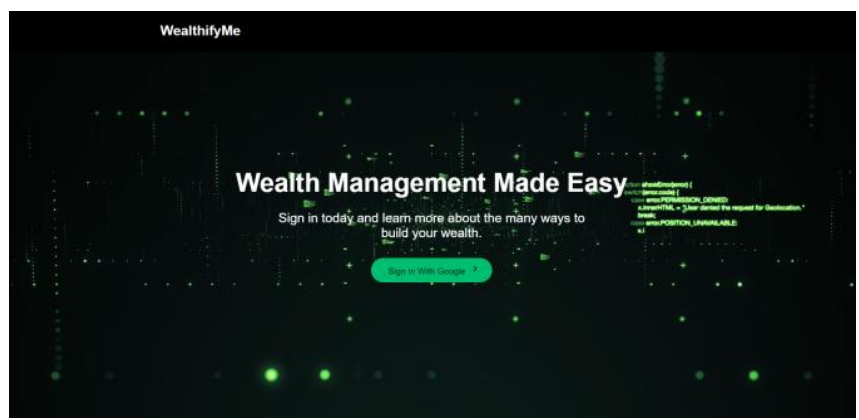

Fig 2: User's First view into the system

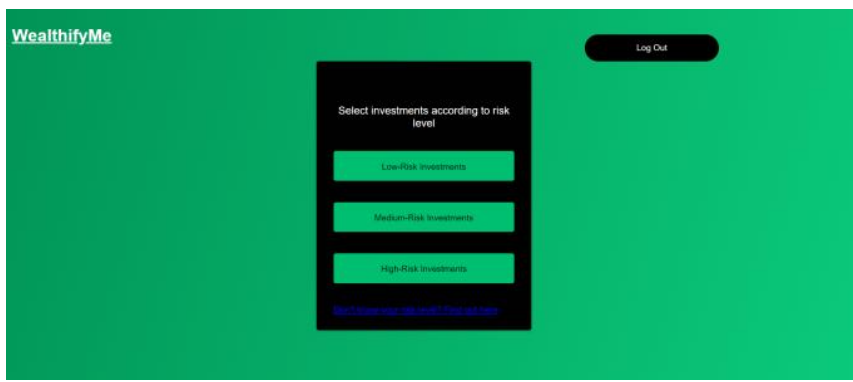

Fig 3: Risk level page of system

\section{B. Customer Segmentation based on Risk Level}

Customer segmentation is a method of categorizing the customers into distinct subgroups with distinct needs. When done manually, it lacks precision and accuracy, and it takes a long time. Artificial intelligence (AI) and machine learning principles are being used in the segmentation process to help you get the most out of your resources and data and achieve your business goals. These 'segments' can be as easy as separating the customers by categories like gender and age, which are the most closely linked to their risk level preferences.

The Customer Segmentation Process are as followed:

- Pre-processing

In order for the process to work, the data must first be cleaned and transformed. It will also be necessary to identify a 'gold standard' training collection for future use.

- Modeling

Algorithms will be run to determine the variables are crucial to segmentation. These are then prioritized and added to the 'gold standard' training set so that the model can learn which properties are shared by the segments.

\section{- Evaluation}

A matrix can be used to distinguish previously incorrectly defined contacts and also be used to determine the model's accuracy. A statistical coefficient can be used to account for class imbalance when the data set includes unbalanced data across segments.

\section{- Output}

The data has now been transformed. The customers will now be segmented based on the "gold standard" training set.
The users have an option where they can find out the risk appetite, they would fall into by simply filling up a form which includes information about their age, gender and certain personality-based questons.

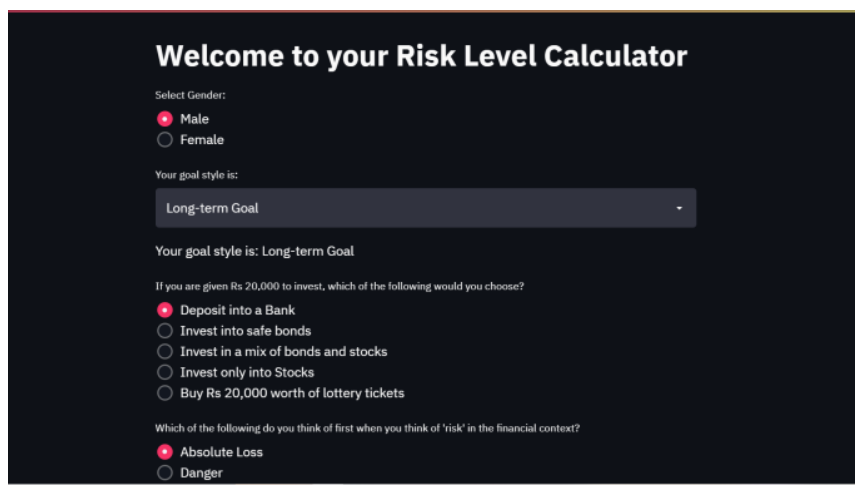

Fig 4: The form to calculate risk

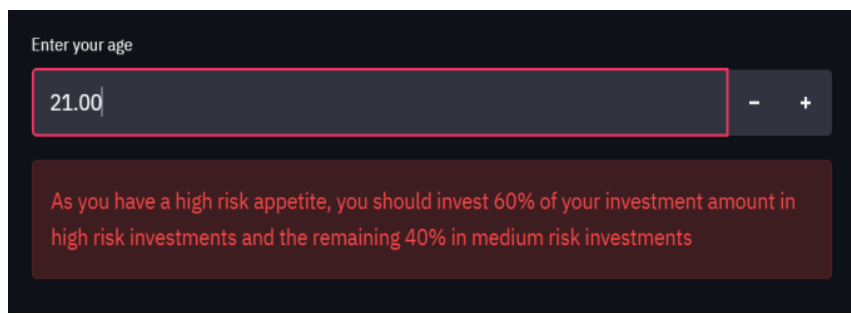

Fig 5: This is the result of a user with high risk appetite

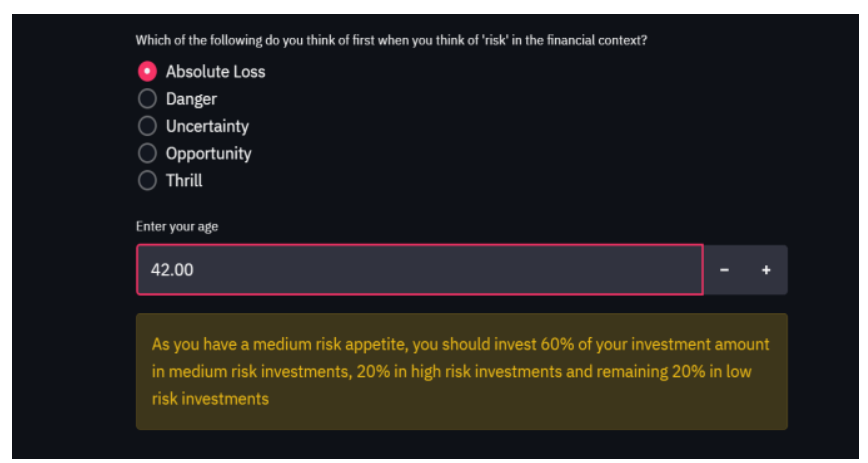

Fig 6: This is the result of a user with medium risk appetite

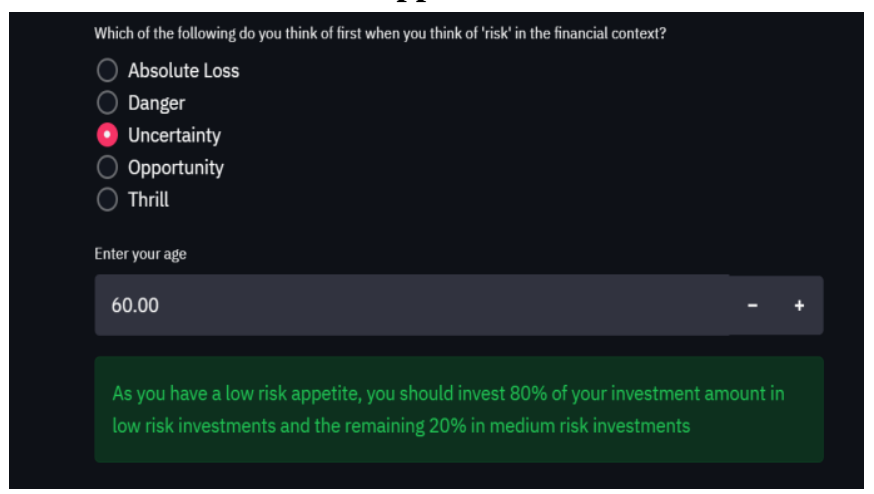

Fig 7: This is the result of a user with low risk appetite

Published By:

Blue Eyes Intelligence Engineering and Sciences Publication

(C) Copyright: All rights reserved.

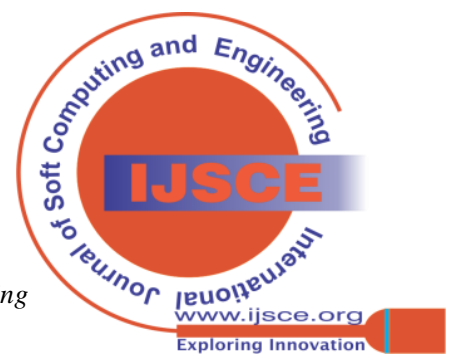




\section{Low-risk and Medium-risk investments (Return calculator)}

We've built a Returns Calculator that can be used to figure out how much money the user will make with the investment that they are looking into. There will be different parts, much like any calculator, where the user can enter the various parameters depending on which the returns on investment will be determined.

\section{High-risk investments (Stock Price Prediction)}

Prediction problems are around for an extended time. They are together considered as the toughest problems to unravel within the data science industry. These include a good range of problems; from predicting sales to finding patterns available markets' data, from understanding movie plots to recognizing your way of speech, from language translations to even predicting the next word on your keyboard. The stock price of today will depend upon:

\section{- The trend}

The trend that the stock has been following within the previous days, maybe a downtrend or an uptrend.

\section{- The worth}

The worth of the stock on the previous day, because many traders compare the stock's previous day price before buying it.

\section{- Factors}

The factors which will affect the worth of the stock for today. This can be a replacement company policy that's being criticized widely, or a drop by the company's profit, or even an unexpected change within the senior leadership of the company.

Prophet is an additive regression model that fits non-linear patterns with annual, weekly, and regular seasonality, as well as the impact of holidays:

- Prophet detects changes in patterns by selecting change points from the data.

- A Fourier series-based yearly seasonal variable

- Dummy variables are used to create a weekly seasonal component.

- A list of important holidays submitted by users.

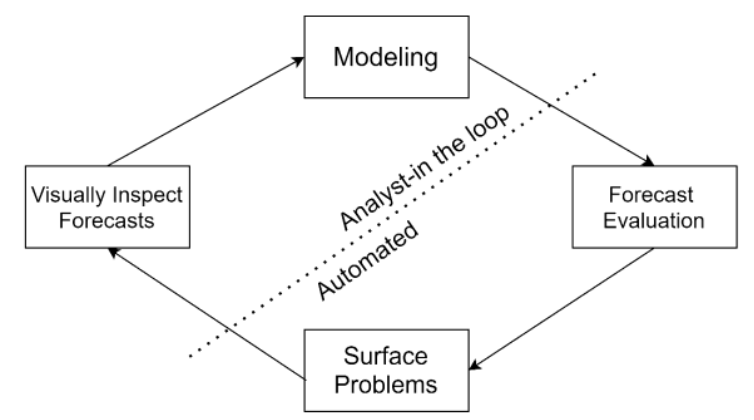

Fig 8: Prediction model of Prophecy used in the Stock Market Prediction

\section{RESULTS AND DISCUSSIONS}

\section{A. Prophecy Library:}

Forecasting is a method of making well-informed forecasts about the path of future developments using historical data as inputs. In today's organizations, it's a critical and popular data science challenge. Knowing of an event in advance will help a company's goal-setting, policy-making, and planning greatly. Prophet is a method designed to overcome these concerns and offers a realistic approach to forecasting "at scale." Its aim is to provide easy and methods which can be tuned for automating the popular features of business time series.

Prophet allows analysts from a wide range of backgrounds to make more predictions than they could manually.

The Prophet kit includes user-friendly parameters that are simple to adjust. And someone with no prior experience with forecasting models may do this to make accurate forecasts for a number of business problems. They are combined in the following equation:

$$
y(t)=g(t)+s(t)+h(t)+\varepsilon_{t}
$$

$g(t)$ : piecewise linear or logistic growth curve for modelling non-periodic changes in time series

$s(t)$ : periodic changes (e.g: weekly/yearly seasonality)

$h(t)$ : effects of holidays with irregular schedules

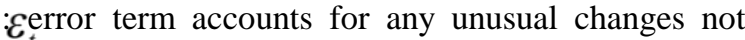
accommodated by the model

The Prophet uses a decomposable time series model. The Prophet procedure is an additive regression model with five main parts:

Prophet automatically detects changes in trends by selecting

changepoints from the data.

A yearly seasonal component modeled using Fourier series.

A weekly seasonal component using dummy variables.

A user-provided list of important holidays.

We sampled this model over Reliance stock to ascertain the prediction and the error percentage. It also had a root mean square error of 1.2

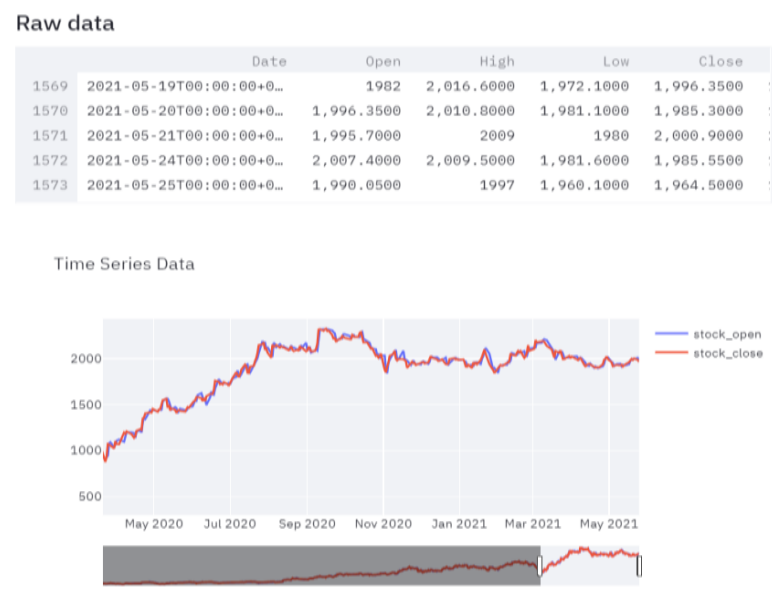

Fig 9: Raw data and graphed Reliance.BO Stock

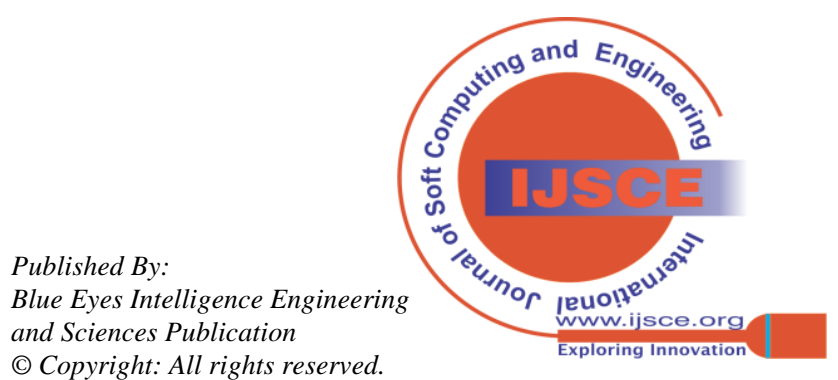



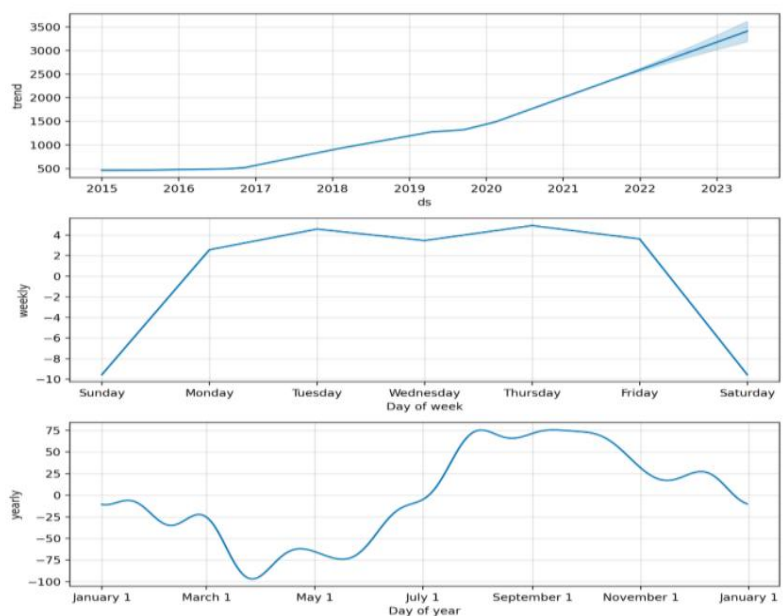

Fig 10: Forecast trends, weekly and yearly for Reliance.BO Stock using Prophecy

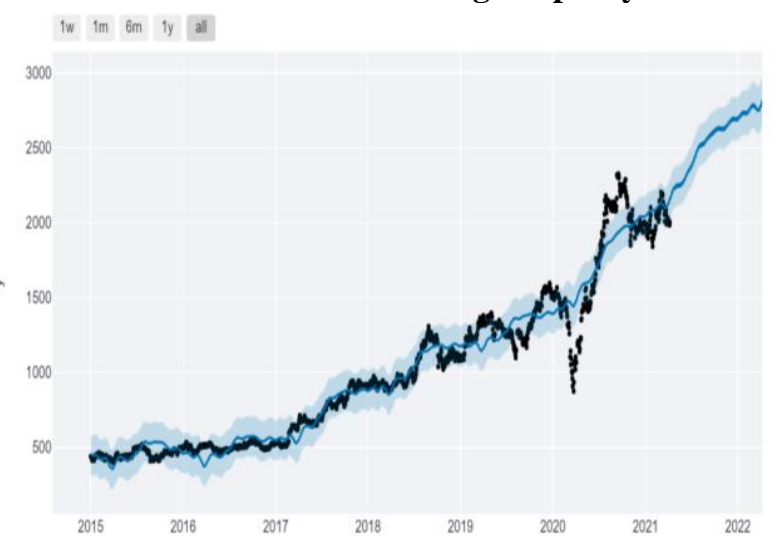

Fig 11: Prediction graph of Reliance.BO Stock using Prophecy

\section{B. LSTM:}

We had also used LSTM neural network to predict the closing price of Apple and Netflix stock using Yahoo finance information. This model are often wont to predict the stock of any company. Just by replacing the company name in the code.

Prediction problems are around for an extended time. They are considered together of the toughest problems to unravel within the data science industry. These include a good range of problems; from predicting sales to finding patterns available markets' data, from understanding movie plots to recognizing an individual's way of speech, from language translations to predicting the next word. With the recent breakthroughs that are happening in data science, it's found that for nearly all of those sequence prediction problems, long short-Term Memory networks, a.k.a LSTMs are observed because the best solution.

LSTMs have a certain edge over conventional feed-forward neural networks and Recurrent Neural Network (RNN) in many ways. They have the unique feature of selectively remembering patterns for long durations of your time.

LSTMs can selectively remember or forget things. The information at a specific cell state has three different dependencies. We'll visualize this with an example. Let's take the instance of predicting stock prices for a specific stock.

The stock price of today will depend upon:

The trend that the stock has followed in the previous days, the worth of the stock on the previous day, because many traders compare the stock's previous day price before buying it.

Factors which will affect the price of the stock for today.

This can be a replacement company policy that's being criticized widely, or a drop by the company's profit, or even an unexpected change within the senior leadership of the company.

These dependencies are often generalized to any problem as:

The previous cell states

The previous hidden state

The input at the present time step

We calculated a rmse of $4.7 \%$

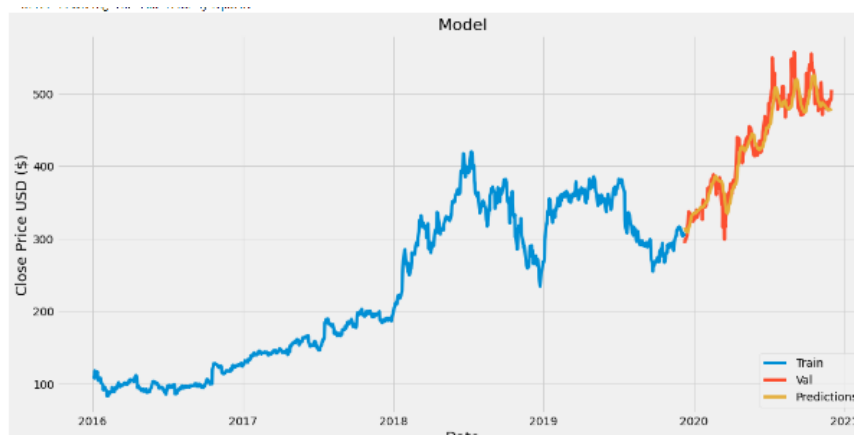

Fig 12: Stock price prediction graph of NETFLIX

We have tried the various algorithms and referred to a number of papers who have focused on the particular algorithms to ascertain the aspects that need to be focused on while choosing the algorithm to work with on the Stock price

\begin{tabular}{|l|l|l|l|}
\hline $\begin{array}{l}\text { Algorithm } \\
\text { Used }\end{array}$ & $\begin{array}{l}\text { Model } \\
\text { Applied }\end{array}$ & Parameters & Metrics \\
\hline SVM [3] & $\begin{array}{l}\text { Non-Linear } \\
\text { Classification }\end{array}$ & Accuracy & $96.15 \%$ \\
\hline $\begin{array}{l}\text { NN } \\
\text { (Neural } \\
\text { Network) } \\
\text { [6] }\end{array}$ & & Accuracy & $88 \%$ \\
\hline Prophecy & - & RMSE & 1.2 \\
\hline $\begin{array}{l}\text { LR [2] } \\
\text { Classification }\end{array}$ & RMSE & $\begin{array}{l}1.4 \\
\text { (with } \\
\text { PCA) }\end{array}$ \\
\hline SVM [4] & Regression & RMSE & 12.873 \\
\hline
\end{tabular}

Fig 13: Comparison Table of the various machine learning algorithms

\section{CONCLUSION AND FUTURE SCOPE}

Based on the analysis with various machine learning algorithms, we have found Prophecy to be the best suited to the need of this system. We used clustering techniques to work on the customer segregation based on risk level and have used react for the website implementation for a smooth and interactive view of the user.

Published By:

Blue Eyes Intelligence Engineering and Sciences Publication

(C) Copyright: All rights reserved.

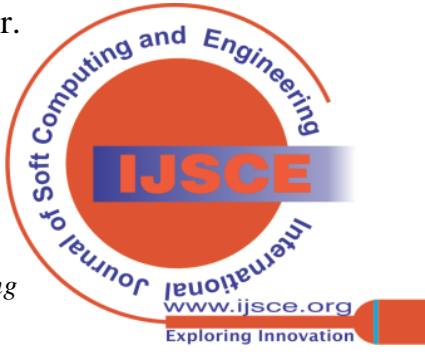


Through this paper, we have built a wealth management system which uses machine learning and artificial intelligence principles to help the users make financially sound decisions. This will be a highly reliable and efficient system for modern wealth management industry and market requirements, with aspects of growth in the taxation field.

\section{ACKNOWLEDGMENT}

We have a great pleasure to express our gratitude to all those who have contributed and motivated us during our project work. For selecting a topic of our choice and studying and understanding it, is a good opportunity to learn and know new technology, understand the way projects are researched and executed on international level. We would like to thank our guide Prof. Mohan Bonde for his support and guidance, our HOD Dr.Sanjay Shitole, our principal Dr.Sanjay Pawar, and to all the teachers whose help made us understand our topic well.

Thank you all!

\section{REFERENCES}

1. Ibrahim M. Hamed, Ashraf S. Hussein, Mohamed F. Tolba, An intelligent model for stock market prediction, in the International Journal of Computational Intelligence Systems, 2012

2. P. Guo, M. Waqar, H. Dawood, M. B Shahnawaz and M. A. Ghazanfar, Prediction of Stock Market by Principle Component Analysis in $13^{\text {th }}$ International Conference on Computational Intelligence and Security, 2017

3. Z. Hu, J. Zhu and K. Tse, Stocks Market Prediction Using Support Vector Machine, in 6th International Conference on Information Management, Innovation Management and Industrial Engineering, 2013.

4. S. Kavitha, S. Varuna and R. Ramya, A Comparative Analysis on Linear Regression and Support Vector Regression, in Online International Conference on Green Engineering and Technologies (IC-GET), 2016.

5. Eric Alexander, Emily Kawaler, Dan Szafir, Stock Market Analysis and Prediction, 2011

6. R. Verma, P. Choure and U. Singh, Neural Networks through Stock Market Data Prediction, in International Conference on Electronics, Communication and Aerospace Technology, 2017.

7. Meghna Misra1, Ajay Prakash Yadav, Harkiran Kaur, Stock Market Analysis and Prediction, In International Conference on Recent Innovations in Electrical, Electronics and Communication Engineering (ICRIEECE), 2018

8. Prakhar Vats, Krishna Samdani, Study on Machine Learning Techniques in Financial Markets, IEEE International Conference on System, Computation, Automation and Networking (ICSCAN), 2019

9. Hirotaka Mizuno, Michitaka Kosaka, Hiroshi Yajima Application of Neural Network to Technical Analysis of Stock Market Prediction, 2001

10. Ayodele A. Adebiyi., Aderemi O. Adewumi, Charles K. Ayo, Stock Price Prediction Using the ARIMA Model, in UKSim-AMSS 16th International Conference on Computer Modelling and Simulation, 2014

11. Samarth Agarwal, Manoj Jindal, G>N Pillai, Momentum Analysis based Stock Market Prediction using Adaptive Neuro-Fuzzy Inference System, in International Multi-Conference of Engineers and Computer Scientists 2010 Vol I, IMECS, 2010

12. Qianwen Bi, Jingpeng Tang, Bradley Van Fleet, Software Architecture for Machine Learning in Personal Financial Planning, In Intermountain Engineering, Technology and Computing (IETC), 2020

13. V. Rajput and S. Bobde, Stock Market Prediction Using Hybrid Approach, in International Conference on Computing, Communication and Automation (ICCCA2016), 2016.

14. R. Verma, P. Choure and U. Singh, Neural Networks through Stock Market Data Prediction, in International Conference on Electronics, Communication and Aerospace Technology, 2017.

15. Brenda Y. Hon, Dickson K.w. Chiu, An Integration of Web Service and Workflow to a Wealth Management Order Placement System: A
Case Study of International Brokerages, In IEEE Asia-Pacific

Conference on Services Computing, 2006. (APSCC'06)

16. Finbingo, an online portal, Available at https://www.finbingo.com/

\section{AUTHORS PROFILE}

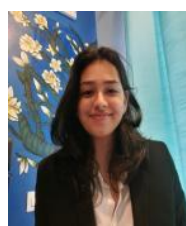

Prarthana Mukherjee, is currently pursuing Bachelor of Engineering degree in Information Technology from Usha Mittal Institute of Technology, SNDT Women's University, Mumbai, Maharashtra, India. Her area of interest includes Machine Learning, Artificial Intelligence and Big Data.

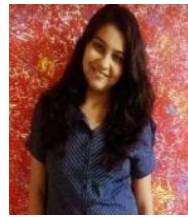

Prit Palan, is currently pursuing Bachelor of Engineering degree in Information Technology from Usha Mittal Institute of Technology, SNDT Women's University, Mumbai, Maharashtra, India. Her area of interest includes Machine Learning, Artificial Intelligence and Cloud Computing.

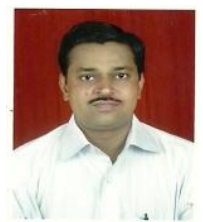

M. V. Bonde, has obtained Bachelor of Engineering degree in Computer Science \& Engineering from Dr. B. A. Marathwada University, Aurangabad, Maharashtra, India in 1997. He completed Master of Engineering in Computer Engineering from Mumbai University, Mumbai, Maharashtra, India in 2003. He is currently working as Assistant Professor in Information Technology Department at Usha Mittal Institute of Technology, SNDT Women's University, Mumbai, Maharashtra, India since 2003. He has published 8 research papers in International Journals. His main areas of interest include Digital Signal and Image Processing, Information Security. He has 19 years of teaching experience and 2 years of industry experience.
Published By: Blue Eyes Intelligence Engineering and Sciences Publication (c) Copyright: All rights reserved.

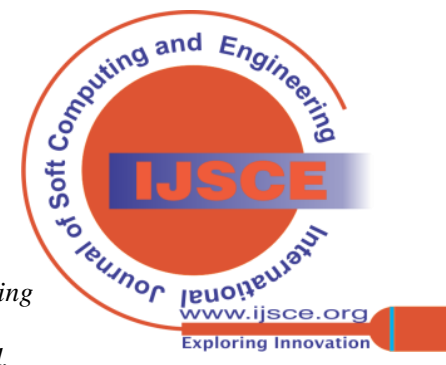

